\title{
How Can Citizens Be Informed: Examining the Democratic Practices of iVoters
}

\author{
Prof. Da-Chi Liao \\ Professor of the Institute of Political Science, National Sun Yat-Sen University. \\ (dcliao@mail.nsysu.edu.tw)
}

Keywords: Questionnaires, iVoter, Deliberative democracy.

\begin{abstract}
Democracy is experiencing it is worst setback since the mid-20th Century. The US magazine Foreign Affairs even posed the question in an article entitled "Is Democracy Dying?" Published on April 2018 issue, assessing the impact that exogenous factors have on liberal democracy. The exogenous factors is the rise of European political parties and American conservatives because the cross-border flow of migrants and refugees and the blurring of boundaries due the rise of China and globalization. This paper examines how citizens acquire information of political issues, whether they can succesfully acquire such knowledge, the effect of information on citizen political decision-making, and problems in Taiwan, particularly sociologist, have focused on the deliberative democracy in Taiwan, particulary sociologist have focused on deliberative procces concerning local public issues that are of concern to resident.
\end{abstract}

\section{INTRODUCTION}

Deviations of democratic practices from ideal paradigm are an irrefutable fact. The ideal paradigm consist of acquiring knowledge before voting for a political representative or policy and is the fundamental starting point envisaged by classical democracy theory.This ideal paradigm consist of acquiring knowledge before voting for a political representative or policy and it is the fundamental starting point envisaged by classical democratic theory. This ideal subject to the paradox of empirical democracy theory, but it is valued and promoted by deliberative democracy theorist. Deliberative democracy needs people or society to looking at private selfinterest that have been provided in the community. This private self-interest in the term of deliberative democracy is often called 'the market'. Supporters of deliberative democracy in Taiwan, particularly sociologist, have focused on the deliberative process concerning local public issues that are of concern to resident. This is also another term in deliberative democracy which is called 'forum'.

The experience shared in this paper, based on a political science perspective of election in democracies, is presented in order to describe and examine the origins, practices, benefits, problems, and future expectations of using the online platform "iVoter" in promoting the acquisition of political information by citizens before national elections.

\section{METHODS}

Mix-Method research used to write this research because the long term periode this research itself. This research was conducted since 2000s to find out the political symptoms in society. Qualitative research used to analyze political interest and self-interest in society that channeled through internet. From their vote in the internet, the researcher can also use quantitative methods from the questionnaires made by iVoters. These web iVoters made a pattern that can analyze simultaneously. Based on a political science perspective of election in democracies, is presented in order to describe and examines the phenomena clearly and completely through web. 


\section{RESULT AND DISCUSSION}

Since 2010, the authors has been developing the iVoters (http://ivoter.tw/) (Wu-Nan Book,2013) with the support of the Chiang Ching-Kuo Foundation and Alexander $\mathrm{H}$. Treschel, a professor of political science from the European University Institute. The website provides voters with information on the issue positions of each candidate before a legislative election. Such information is obtained through questionnaires completed by candidates and political parties (iVoter, 2013). To understand more about the uses of iVoters, the researchers will show the table about questionnaries that already analyzed and organized as illustrated below :
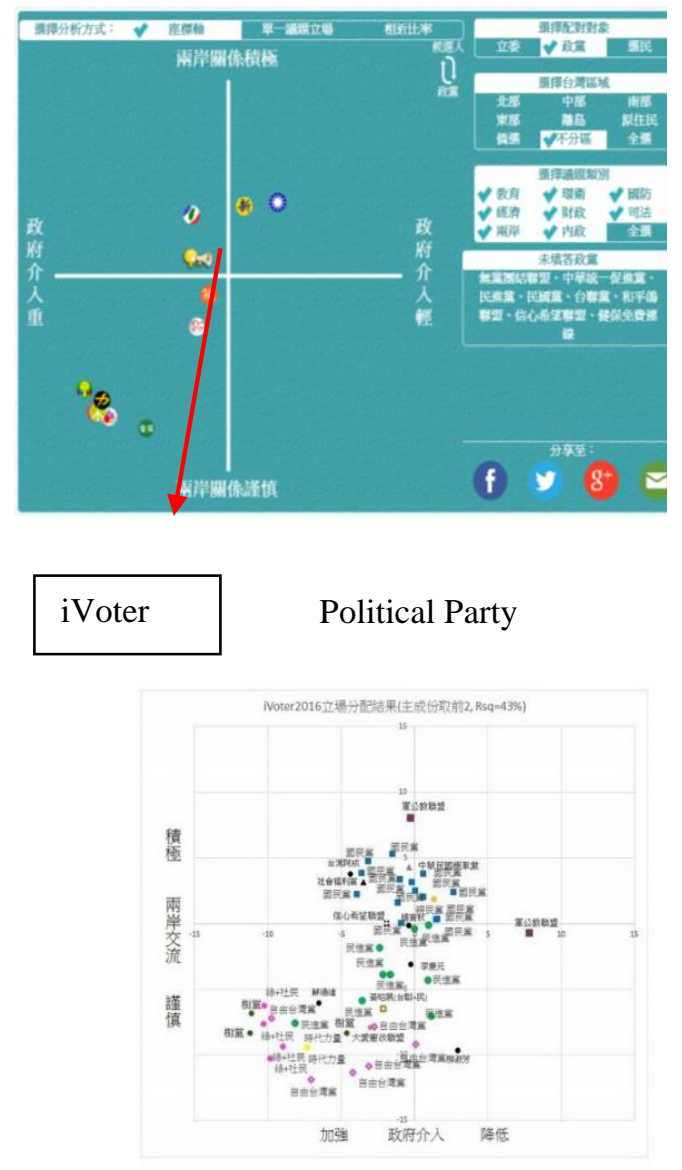

Candidate

Figure 1. Distribution

and

Coordinates axes illustrating the positions of political parties and candidates on various issues in 2016 iVoters political spectrum test.

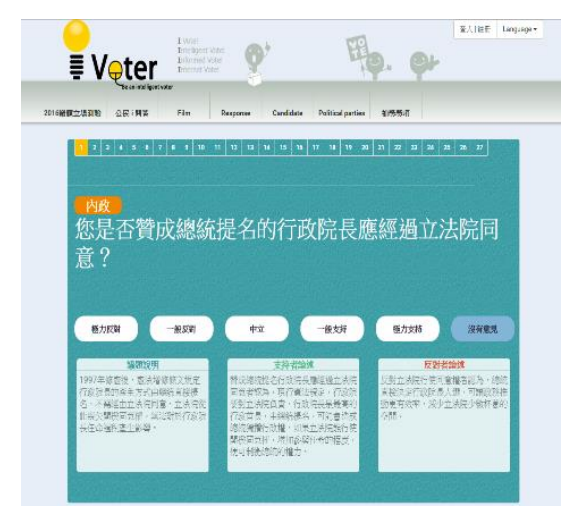

Figure 2. Short explanation of each questions.
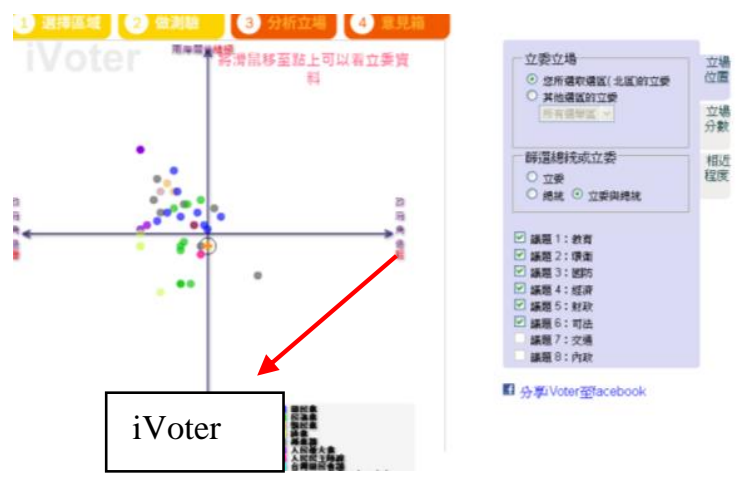

Figure 3. Matching of the two-dimensional distribution of the position of an iVoter respondend and candidates on issues in 2012.

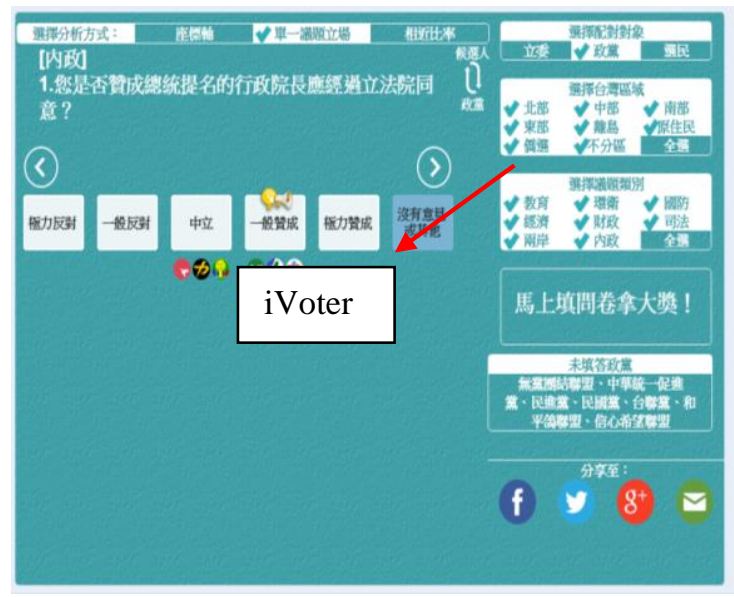

Figure 4. Position of an iVoter repondend and political parties on a single issue. 


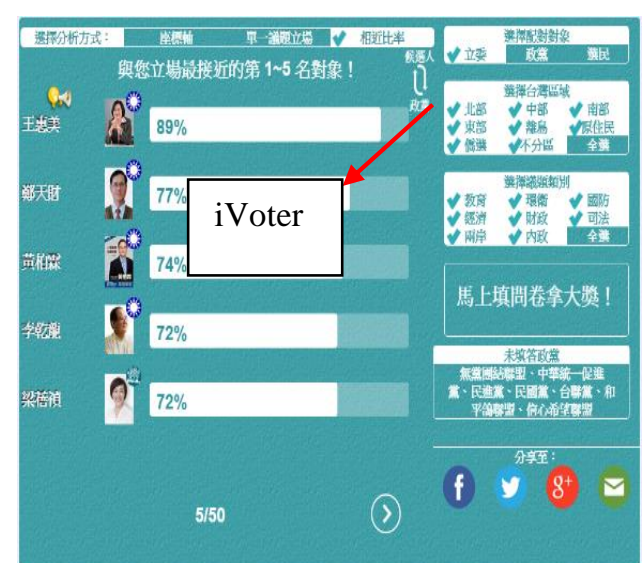

Figure 5. Percentage similarity between all issue positions of a candidate and an iVoter respondend

As we can see in the figure above, in the figure 1 voters are invited to complete the same questionnaire on the iVoter website, the only things that make this figure difference is that issues and assenting or dissenting opinions by voters are presented on the webpage, providing voters with more reference information as we can see in the figure 2 which the use is to determine how their policy stances match with those candidates or parties in the figure 3-5. In the figure above also shows that internet has a very large role in determining the interest of people voiced through iVoters.

'Quick Count' phenomena can also be done through iVoters. Ultimately, iVoter aim to help voters obtain customized information on candidates or parties whose political stances match their own preferences before the election. Therefore, the design of iVoter is called the voting advice application (VAA) in English-language literature, and the "I" in the name iVoter implies being an informed, intelligent, and Internet-savvy voter. In this paper, iVoter is used to refer to both the Taiwanese VAA and the respondent who used the iVoter website before the 2012 and 2016 legislative elections in Taiwan.

The analysis covered 21 issues for 2012 and 27 issues for 2016 as can see figure 1 depict the factor analysis of all issues. The horizontal axis represents the traditional left to right political spectrum and positions on the axis are indicated by the level of government intervention from deepest to lightest ends. The axis represents the Taiwan Strait concern, which is a polarizing political domain among electorate in Taiwan. The authors and teams assessed views with respect to "Cautiousness or cordiality in cross-strait relations" rather than posing a stronger binary opposition (reunification vs independence) for this domain. Figure 2 illustrates an interface design that is intended to help voters quickly acquire information regarding each issue. Figures 1 and 3-5 demonstrate that iVoters can use various approaches to ascertain which candidate or political party most closely matches their political stances. Figure 1 represents the position of a political party, Figure 3 represents the position of all candidates and a certain iVoter on the two dimentional-axes, Figure 4 represents an iVoter's match with the stance of a political party or candidate on a specific issue, and Figure 5 presents the similarity between the positions of iVoters and candidates or political parties on all of the issues selected.

The iVoter website was launched before the two major elections in 2012 and 2016 and received considerable support. The website amassed more than 40,000 users in 2012. Approximately 1,400 users completed our pre-election questionnaire (designed for research purposes and ascertaining political stances) and 647 users completed the postelection questionnaire, making iVoter the most effective platform among all VAAs worldwide for generating data for research use. VAA is most frequently used in European countries, the earliest being in the Netherlands on 1989. The number of iVoter users increased in 2016, the platform reached 85,000 views, and 2,474 questionnaires were retrieved.

We assessed whether the political spectrum on the iVoter website was helpful by analyzing the concept "helpful" in two respects: "knowing" and "acting." "Knowing" refers to whether a piece of information is worth considering when deciding how to vote. "Acting" refers to voter behavior in which the voter changes her initial choice because of a particular piece of information (because the voter now understands that the originally supported candidate or party does not share the voter's political beliefs) and in turn votes for a candidate or party that holds political positions in line with the voter's own. The effect of "knowing" on voter behavior is closely in line with the expectations of classical democracy theory.

iVoter demonstrated a significant effect on user knowledge. Question items such as "did [the information] help you to understand the positions of your legislator (or political party) on an issue" or statements such as "the political spectrum test result helped me to decide my vote" were included in the 2012 and 2016 questionnaires, and approximately 
$70 \%$ of the respondents answered in the affirmative. In addition, the results also indicated that iVoter is probably capable of inducing users to continue seeking information concerning political parties and candidates whose positions are similar to theirs. In the 2016 questionnaire, we asked whether respondents wished to acquire further information concerning legislators (or political parties) who share their political positions, and affirmative responses were obtained from $80 \%$ of the respondents on average, which is higher than the number of respondents indicated above $(70 \%)$. These results suggest that the indirect effect of iVoter is apparently stronger than its direct effect.

We also attempted a preliminary exploration of the transition from "knowing" to "acting." The administration of two rounds (one before and one after election) of questionnaires was arranged in 2012, and a kind of panel design was used to track the changes in the voting decisions of iVoters who were willing to complete the two rounds of questionnaires concerning their use of the iVoter platform. One of our team members (Hung-En Wang, 2012), even published a paper on this topic entitled "Information Utility of iVoter."(iVoters.2013).

According to Wang's analysis, a high percentage of voters tended to change their votes after using the iVoter website and a majority of these voters shifted their support to small political parties or candidates from bigger political parties (iVoters,2013). In 2016, we did not administer two rounds of questionnaires (one before and one after election). Instead, a round of questionnaires was administered before the election just after using the iVoter website, because the direct causal relationship between iVoter information and voting behavior was difficult to determine even when a panel design was used.

Therefore, the design was changed to incorporate an experimental design in the interest of verifying the direct effect of iVoter information. Preliminary findings verified that the interactive function of the iVoter website helped users to collect relevant information and increased the intention to vote among users. However, whether such information that helps users to know their views and those of candidates and political parties influences voters' decisions remains unknown. The democracypromoting tool iVoter has been implemented for more than eight years. User feedback and questionnaire responses have in general indicated a positive recognition of the iVoter platform, especially its method and promotion of political information among citizens.

Nevertheless, the website has several problems that warrant examination to discern aspects that require improvement. The first problem concerns issue selection and the method and direction for compiling a discourse for each issue. Our current approach is to conduct text mining of the data of the Legislative Yuan and media data to achieve topic detection and tracking (TDT), then select issues on the basis of the professional judgment of our research team. Issue content and assenting/dissenting opinions are compiled primarily from information associated with topics extracted using TDT or controversy discussed in media reports (Hung-En Wang,2012).

Although we aimed to be objective, intellectuals were inevitably curious and skeptical when the author was promoting the iVoter website. We also restricted the amount of information presented on the website to secure users' willingness to "know"; therefore, not everything could be covered in each explanation. The second problem concerns the reliability of issue positions expressed by candidates and political parties.

Despite undertaking the utmost effort to acquire such information, pieces of information were still missing. In 2012, 92 candidates (including candidates not running for office at that time) had completed the questionnaire, but only 53 of them did so again in 2016. A section on political party was incorporated in 2016 that involved interviews with each political party. The information collected was registered only after it was confirmed by the party chair. Among the 18 participating political parties nationwide, 12 parties completed the questionnaire, which was a notable response rate (.). Workers of the Democratic Progressive Party, which was the expected winning party at the time, completed the questionnaire with reference to their campaign pledges.

The questionnaires were sent to the highest governing body, which prohibited disclosure of questionnaire results. Our team respected that mandate, although we thought it was a pity to conceal such valuable information from the public. Were the responses of each political party reliable? From the author's perspective, because "neutral" was one of the options for responding to the questionnaire, party respondents could avoid responding to sensitive issues. 
However, they clearly indicated their positions when the positions were highlighted in their party program. Because this collected information is disclosed on the website, political parties most likely responded with the utmost care. The third problem is the penetration of iVoter among prospective voters. The average age of iVoter users is approximately 26 years, and most of the users are supporters of small political parties. The number of users is calculated to be only 80,000 based on the highest number of views, which raises the question whether iVoter can encourage citizens to seek information. The fourth problem concerns the scope of iVoter, which provides information on issues before an election-perhaps the scope of the service should be expanded to periods between elections to better achieve its goal of promoting a politically savvy citizenry.

We have formulated a few preliminary ideas and adopted some approaches to address the aforementioned problems. These ideas are briefly described below. In response to the first problem, we thought of the possibility of using crowdsourcing to select and compile issues.

Regarding the second problem, forging more ties with NGO groups (e.g., the Citizen Congress Watch) might motivate candidates and parties to complete the political spectrum test questionnaire. Concerning the third problem, we contemplated whether a VAA such as iVoter can act as an information provider for government departments (e.g., election commission). For instance, the VAA was widely adopted only after the Dutch and German governments incorporated the application into the country's mechanism for promoting civic education. The application has amassed millions of users in both countries. With respect to the fourth problem, the iVoter team administered the 2016 political spectrum test to high school students in a civics class during a nonelection period and generally received positive responses from students that supported and promoted by National Tainan First Senior High School and The Taipei Municipal Dazhi High School.

In fact, before the political preferences of younger generations ossify, increasing their access to political information and fostering the habit of supporting a party or candidate based on their position on an issue are perhaps key methods for strengthening democracy.

\section{CONCLUSION}

In conclusion, the implication of the iVoter tool regarding strengthening democracy is associated with two positive aspects, and a negative one which is an inexplicable human phenomenon. The first positive aspect is that citizens' thirst for information can be inspired and cultivated, and the second aspect is that young adults of the information age can become paragons of informed citizens. Their knowledge and willingness to acquire information are probably a long-term driving force for strengthening democracy.

The inexplicable aspect is that described by Downs in his books An Economic Theory of Democracy (Downs,Antony. 1957) making a judgment based on information is inevitably in the nature of a rational person but any rational person is just as likely to dismiss or ignore information when it is excessive or overly complicated. Therefore, efforts must be undertaken using various methods to determine how much information to provide and how to provide such information.

\section{REFERENCES}

Wu-Nan Book Inc. Internet Democracy: Records of Establishing the iVoter Consulting Website. 2013.

Downs,Anthony. An Economic Theory of Democracy. From New York: Harper \& Row. 1957

Ivoters. 2013. 